\title{
HYDRODYNAMIC PROBLEMS ARISING FROM THE INVESTIGATION OF THE TRANSVERSE CIRCULATION IN THE ATMOSPHERE
}

\author{
H. J. STEWART
}

Introduction. The motion of the atmosphere can be considered as a mean flow which has a very large scale and is only slowly changed and, superimposed on this, the low level, smaller scale phenomena usually associated with the polar front. If the mean pressures over a period of about a week are plotted, it is seen that the latter disturbances are averaged out and only the large scale mean motion is shown. Such a plot of the northern hemisphere shows, in addition to the mean westerly flow of air, large scale closed isobaric systems spaced at comparatively regular intervals over the surface of the earth. These include the Aleutian and Icelandic low pressure areas to the north of the westerlies and the Pacific and Bermuda high pressure areas to the south of the westerlies.

It has been noticed that the position and strength of these systems control the paths of the low level storms. This fact has been used in the development of a long range forecasting technique which has proved to be very successful for periods as long as three months. A knowledge of the properties of these large scale systems is thus not only of academic interest but of considerable value in the development of long range forecasting techniques.

In the investigation of these large scale transverse motions in the atmosphere, two controlling factors have been suggested and discussed by the author [1] and by Rossby [2] and Haurwitz [3]. These are first, the dynamical instability of the shearing motion on either side of the belt of westerly winds and second, certain forced oscillations in the atmosphere. The first of these and several related problems are discussed here.

In making these calculations several approximations are made. The principal ones and the reasons for their adoption are as follows:

(1) As the systems are very deep, that is, the wind momentum vector for a vertical section is roughly constant, it was felt that the horizontal field of motion was the dominant factor; consequently, vertical velocities are neglected; and horizontal momentum is assumed not to vary with height.

An address delivered before the meeting of the Society in Stanford University, Calif., April 5, 1941, by invitation of the Program Committee; received by the editors August 24, 1942. 
(2) With this dynamical setup, the effect of variation of density with altitude would probably be small; consequently the atmosphere is treated as a layer of fluid of constant density with the depth being determined by the hydrostatic law.

(3) All frictional effects are neglected.

(4) In most of these calculations it is assumed that the fluid can be considered as being on a rotating disc rather than on a rotating sphere. As on the earth, the gravitational field is assumed to be deformed by an amount large enough to cancel out the centrifugal acceleration acting on a particle which is stationary with respect to the rotating disc or sphere.

In the analysis the following notation is used:

$x, y=$ rectangular coordinates on a rotating disc,

$r, \theta=$ polar coordinates on a rotating disc,

$u=$ velocity in the $x$ direction,

$v=$ velocity in the $y$ direction,

$v_{\theta}=$ velocity in the tangential direction,

$h=$ depth of fluid layer,

$\omega=$ angular velocity of the disc,

$g=$ acceleration due to gravity.

The fundamental relations. Using the assumptions discussed in the preceding section, the equations determining the fluid motion on a rotating disc (see $[4$, p. 317]) are, for a Cartesian coordinate system, the dynamical equations,

$$
\frac{D u}{D t}-2 \omega v=-g \frac{\partial h}{\partial x}
$$

and

$$
\frac{D v}{D t}+2 \omega u=-g \frac{\partial h}{\partial y},
$$

and the equation of continuity,

$$
\frac{D h}{D t}+h\left\{\frac{\partial u}{\partial x}+\frac{\partial v}{\partial y}\right\}=0
$$

where $D() / D t$ is the derivative following the fluid particle. If $h$ is eliminated from (1) and (2) by cross-differentiation, the result may be combined with (3) to obtain

$$
\frac{D}{D t}\left\{\frac{\zeta+2 \omega}{h}\right\}=0
$$


where

$$
\zeta=\frac{\partial v}{\partial x}-\frac{\partial u}{\partial y} .
$$

This is the law of conservation of vorticity and could just as well have been taken as one of the fundamental equations. If the motion of the fluid could have been started from rest with a uniform depth, $h_{0}$, the last equation can be integrated and the result obtained that

$$
\frac{\zeta+2 \omega}{h}=\frac{2 \omega}{h_{0}} .
$$

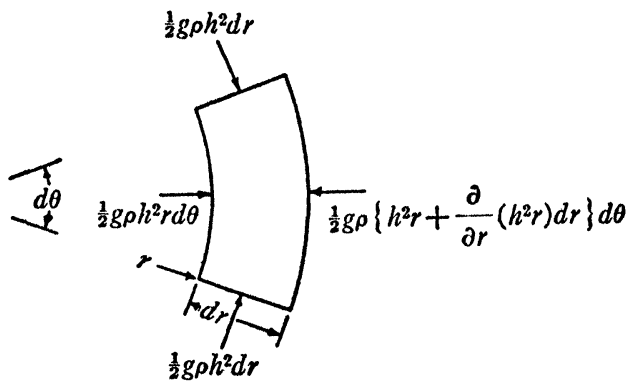

FIg. 1

Diagram of forces for flow in circles

As the dynamical equations are nonlinear, general solutions to these expressions are not readily found; however, the solution for the case of steady motion in circles can be obtained quite easily. The equilibrium of forces in the radial direction (see Figure 1) requires that

$$
(1 / 2) g r \frac{d}{d r}\left(h^{2}\right)=h v_{\theta}^{2}+2 \omega r h v_{\theta} .
$$

From (6),

$$
h=h_{0}\{1+\zeta / 2 \omega\}=h_{0}\left\{1+\frac{1}{2 \omega r} \frac{d}{d r}\left(r v_{\theta}\right)\right\} .
$$

The equation which determines the velocity is thus

$$
\frac{d^{2} v_{\theta}}{d r^{2}}+\frac{1}{r} \frac{d v_{\theta}}{d r}-\left\{\frac{4 \omega^{2}}{g h_{0}}+\frac{1}{r^{2}}\right\} v_{\theta}=\frac{2 \omega}{g h_{0} r} v_{\theta}^{2} .
$$


This can be put into a somewhat simpler form by using the dimensionless variables, $\rho=2 \omega r /\left(g h_{0}\right)^{1 / 2}$ and $V_{\theta}=v_{\theta} /\left(g h_{0}\right)^{1 / 2}$. With these variables, (9) becomes

$$
\frac{d^{2} V_{\theta}}{d \rho^{2}}+\frac{1}{\rho} \frac{d V_{\theta}}{d \rho}-\left\{1+\frac{1}{\rho^{2}}\right\} V_{\theta}=\frac{1}{\rho} V_{\theta}^{2} .
$$

The desired solutions of this equation are those which vanish at infinity and thus correspond to vortex motion. If $V_{\theta}$ is positive, this is the equation for cyclonic rotation; if $V_{\theta}$ is negative, this is the equation for anticyclonic rotation. These solutions were found numerically at the Massachusetts Institute of Technology by means of the Differential Analyzer. These results are plotted in Figure 2

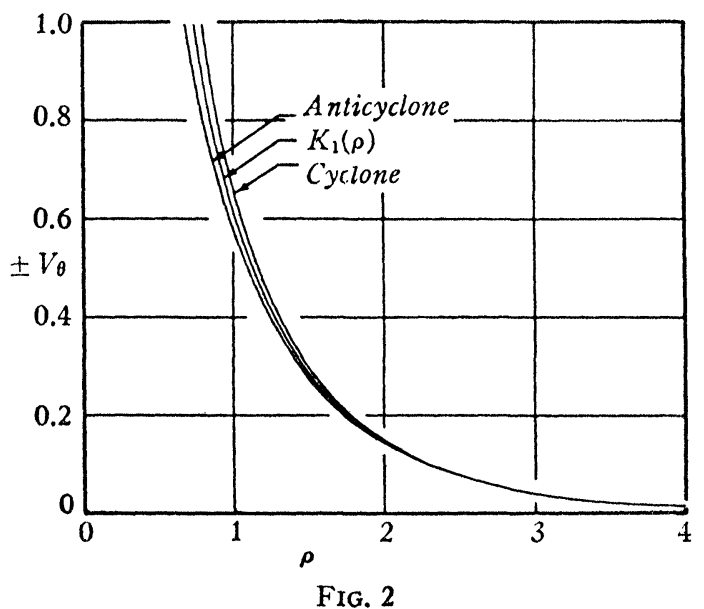

Velocity profiles for vortices

together with $K_{1}(\rho)$ which they approach asymptotically. These results were calculated from this asymptotic solution and thus can be expected to diverge more and more from the true solutions as $\rho$ decreases. From physical considerations it appears improbable that the anticyclonic solution should have the velocity approach negative infinity at the origin. There exists a solution which is finite at the origin and which has a series expansion which starts as follows:

$$
V_{\theta}=a\left\{\rho+\frac{1+a}{8} \rho^{2}+\frac{(1+a)(1+2 a)}{192} \rho^{8}+\cdots\right\} .
$$

It seems probable that the anticyclonic solution must start out this way for some particular value of $a$, but this conjecture has not been verified. 
An approximate solution to this same problem may be found by linearizing the quadratic terms of (1), (2), and (3), that is, by replacing $D() / D t$ by $\partial() / \partial t$. If the linearized equations are solved for $h$, it is seen that

$$
\frac{\partial^{2} h}{\partial t^{2}}-g h_{0}\left\{\frac{\partial^{2} h}{\partial x^{2}}+\frac{\partial^{2} h}{\partial y^{2}}\right\}+4 \omega^{2}\left(h-h_{0}\right)=0 .
$$

In terms of dimensionless variables $X=2 \omega x /\left(g h_{0}\right)^{1 / 2}, Y=2 \omega y /\left(g h_{0}\right)^{1 / 2}$ and $\eta=\left(h-h_{0}\right) / h_{0}$, this equation becomes

$$
\frac{\partial^{2} \eta}{\partial t^{2}}-\left\{\frac{\partial^{2} \eta}{\partial X^{2}}+\frac{\partial^{2} \eta}{\partial Y^{2}}\right\}+\eta=0 .
$$

The only steady state solution to this equation which vanishes at infinity and which represents flow in circles about the origin is

$$
\eta=A K_{0}(\rho)
$$

where $\rho=\left(X^{2}+Y^{2}\right)^{1 / 2}$ is the same as in (10), $A$ is an arbitrary constant, and $K_{0}(\rho)$ is a modified Bessel function of the second type $[5$, p. 21]. From (1), it is seen that

$$
V_{\theta}=\frac{v_{\theta}}{\left(g h_{0}\right)^{1 / 2}}=\frac{\partial \eta}{\partial \rho}=-A K_{1}(\rho) .
$$

This is plotted for a unit cyclone, $A=-1$, in Figure 2 together with the exact solutions which retained the quadratic terms. From (15) it can be seen that if $\rho \ll 1$, the velocity varies inversely as the radius, just as in an ordinary line vortex in an incompressible nonviscous fluid, and if distances between the vortices of (14) are small, results identical to those found with ordinary vortices will be obtained. However, the linearization of the equations of motion breaks down in this range; so that results obtained with ordinary vortices cannot be applied directly.

If $h_{0}$ is taken as being the depth of the homogeneous atmosphere, about $8 \mathrm{~km}$, a distance of about $2,000 \mathrm{~km}$ corresponds to $\rho=1$. Figure 2 or (10) shows that at distances as large as this, the error caused by neglecting the quadratic terms is not large. As the spacing of the pressure centers being investigated is of the order of $2,000 \mathrm{~km}$, the quadratic terms will be neglected throughout, and the linearized form, (13), will be used. In addition to this, the motions will be considered to be either stationary or changing very slowly so that the local acceleration can be neglected as compared to the Coriolis term. This is equivalent to saying that the fluid motions to be considered can be 
built up through superposition of vortices of the type given by (14). With these approximations, (1) and (2) can be written as follows using dimensionless velocities $U=u /\left(g h_{0}\right)^{1 / 2}$ and $V=v /\left(g h_{0}\right)^{1 / 2}$ :

$$
\begin{aligned}
& V=\frac{\partial \eta}{\partial X}, \\
& U=-\frac{\partial \eta}{\partial Y} .
\end{aligned}
$$

These are the well known geostrophic wind equations.

It is of interest to note that (13) is the same as the equation for the deflection of a membrane which is elastically supported. The character of its solutions may often be estimated by use of this analogy.

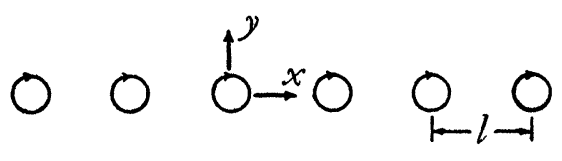

FIG. 3

Single row of vortices

Stability of rows of vortices. The most noticeable feature of the general circulation of the atmosphere is the belt of westerly winds. On both sides of the westerlies where the wind intensity diminishes rapidly there is a region of concentrated vorticity, cyclonic to the north and anticyclonic to the south of the westerlies. As a first approximation to this, the westerlies may be considered as a uniform jet with stationary air masses on either side. Separating the stationary and moving air masses are vortex sheets. That such vortex sheets are unstable has been shown by Pekeris and others. When an unstable vortex sheet breaks up into discrete eddies, the vorticity may either diffuse throughout the fluid mass or, if there is a stable vortex formation, the vorticity may collect in a definite pattern. As an example of this type of motion, the vortex sheets shed from a two-dimensional bluff body break down and then form the well known Kármán "vortex street."

A vortex sheet such as bounds the jet of westerlies can break up into only one system which is in equilibrium. This is a row of equal vortices equally spaced as in Figure 3 . In terms of the dimensionless variables introduced in the preceding section, the surface deflection for such a system of vortices of unit strength is, by (14), 


$$
\eta=\sum_{n=-\infty}^{\infty} K_{0}\left[(x-n l)^{2}+y^{2}\right]^{1 / 2}
$$

If the $n$th vortex is displaced by amounts $\Delta x_{n}$ and $\Delta y_{n}$ in the $x$ and $y$ directions, respectively, the surface deflection in the displaced position is

$$
\eta=\sum_{n=-\infty}^{\infty} K_{0}\left[\left(x-n l-\Delta x_{n}\right)^{2}+\left(y-\Delta y_{n}\right)^{2}\right]^{1 / 2}
$$

The velocity of a vortex is the sum of the velocities induced by all the other vortices. By (16), the velocity of the vortex at the origin is

$$
\begin{aligned}
& \frac{d}{d t}\left(\Delta x_{0}\right)=-\frac{\partial}{\partial y} \sum_{n=-\infty}^{\infty} K_{0}\left[\left(x-n l-\Delta x_{n}\right)^{2}+\left(y-\Delta y_{n}\right)^{2}\right]^{1 / 2}, \\
& \frac{d}{d t}\left(\Delta y_{0}\right)=\frac{\partial}{\partial x} \sum_{n=-\infty}^{\infty} K_{0}\left[\left(x-n l-\Delta x_{n}\right)^{2}+\left(y-\Delta y_{n}\right)^{2}\right]^{1 / 2}
\end{aligned}
$$

where $x$ and $y$ are to be replaced by $\Delta x_{0}$ and $\Delta y_{0}$, respectively, after the differentiation has been performed. The ()$^{\prime}$ on the summation sign indicates that the term for $n=0$ is to be omitted. If the displacements are small, these expressions can be written as

$$
\begin{aligned}
& \frac{d}{d t}\left(\Delta x_{0}\right)=\sum_{n=-\infty}^{\infty} \frac{K_{1}|n l|}{|n l|}\left(\Delta y_{n}-\Delta y_{0}\right), \\
& \frac{d}{d t}\left(\Delta y_{0}\right)=\sum_{n=-\infty}^{\infty}\left\{\frac{K_{1}|n l|}{|n l|}+K_{0}|n l|\right\}\left(\Delta x_{n}-\Delta x_{0}\right) .
\end{aligned}
$$

Similar expressions for the velocities of the other vortices could be written by symmetry. These form a set of simultaneous differential equations which must be solved in order to determine the motion of the vortices.

As the equations for the infinitesimal displacements are linear, any arbitrary perturbation can be represented as a sum of terms of the type $\Delta x_{n}=\Delta x_{0} e^{i n \phi}$ and $\Delta y_{n}=\Delta y_{0} e^{i n \phi}$ where $0 \leqq \phi \leqq 2 \pi$, and the individual harmonics can be investigated separately. From this,

$$
\begin{aligned}
& \frac{d}{d t}\left(\Delta x_{0}\right)=-2 \Delta y_{0} \sum_{n=1}^{\infty} \frac{K_{1}(n l)}{n l}(1-\cos n \phi), \\
& \frac{d}{d t}\left(\Delta y_{0}\right)=-2 \Delta x_{0} \sum_{n=1}^{\infty}\left\{\frac{K_{1}(n l)}{n l}+K_{0}(n l)\right\}(1-\cos n \phi) .
\end{aligned}
$$

If these two equations are combined, it is seen that 


$$
\begin{aligned}
& \frac{d^{2}}{d t^{2}}\left(\Delta x_{0}\right)-\lambda^{2} \Delta x_{0}=0, \\
& \frac{d^{2}}{d t^{2}}\left(\Delta y_{0}\right)-\lambda^{2} \Delta y_{0}=0
\end{aligned}
$$

where

$$
\begin{aligned}
\lambda^{2}= & 4\left\{\sum_{n=1}^{\infty} \frac{K_{1}(n l)}{n l}(1-\cos n \phi)\right\} \\
& \cdot\left\{\sum_{n=1}^{\infty}\left[\frac{K_{1}(n l)}{n l}+K_{0}(n l)\right](1-\cos n \phi)\right\} .
\end{aligned}
$$

From (22) it is seen that any infinitesimal disturbance will grow in

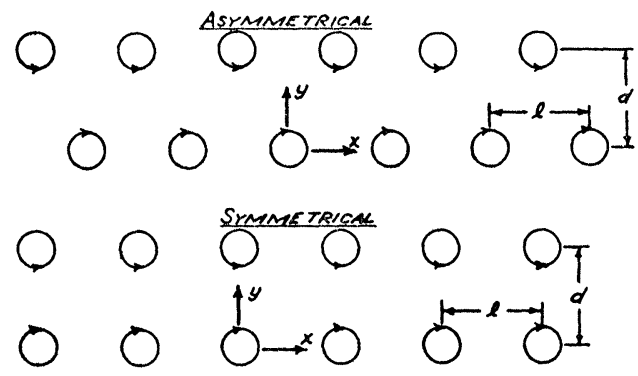

FIG. 4

Double rows of vortices

amplitude unless $\lambda^{2}<0$; however from (23) it is evident that $\lambda^{2}$ is never negative. A single row of vortices is thus unstable.

As there are two shear regions in the northern hemisphere, cyclonic shear to the north of the westerlies and anticyclonic shear to the south, it is possible that a double row of vortices arising from these might be stable. As shown in Figure 4, there are two possible arrangements of equal and opposite vortices which do not change shape with time.

The stability of the symmetrical system will be investigated first. If $\overline{\Delta x}_{n}$ and $\overline{\Delta y}_{n}$ are the displacements of the $n$th vortex in the upper row and $\Delta x_{n}$ and $\Delta y_{n}$ are the displacements of the $n$th vortex in the lower row, the surface deflection is given by

$$
\begin{aligned}
\eta=\sum_{n=-\infty}^{\infty}\left\{K_{0}[(x-\right. & \left.\left.n l-\Delta x_{n}\right)^{2}+\left(y-\Delta y_{n}\right)^{2}\right]^{1 / 2} \\
& \left.-K_{0}\left[\left(x-n l-\overline{\Delta x}_{n}\right)^{2}+\left(y-d-\overline{\Delta y}_{n}\right)^{2}\right]^{1 / 2}\right\} .
\end{aligned}
$$


From this expression the velocities of the vortices can be obtained in the same manner as in the preceding calculation. By writing $\Delta x_{n}=\Delta x_{0} e^{i n \phi}, \Delta y_{n}=\Delta y_{0} e^{i n \phi} \overline{\Delta x}_{n}=\overline{\Delta x}_{0} e^{i n \phi}$ and $\overline{\Delta y}_{n}=\overline{\Delta y}_{0} e^{i n \phi}$ as before, the velocity of the zero vortex in the lower row is seen to be

$$
\begin{aligned}
& \frac{d}{d t}\left(\Delta x_{0}\right)=A \Delta y_{0}+B \overline{\Delta x_{0}}+C \overline{\Delta y_{0}}, \\
& \frac{d}{d t}\left(\Delta y_{0}\right)=D \Delta x_{0}-B \overline{\Delta y_{0}}+E \overline{\Delta x_{0}}
\end{aligned}
$$

where

$$
\begin{aligned}
A= & -K_{0}(d)-\frac{K_{1}(d)}{d}-2 \sum_{n=1}^{\infty}\left\{\frac{K_{1}(n l)}{n l}(1-\cos n \phi)\right. \\
& \left.+d^{2} \frac{K_{0}\left[n^{2} l^{2}+d^{2}\right]^{1 / 2}}{n^{2} l^{2}+d^{2}}-\left(n^{2} l^{2}-d^{2}\right) \frac{K_{1}\left[n^{2} l^{2}+d^{2}\right]^{1 / 2}}{\left[n^{2} l^{2}+d^{2}\right]^{3 / 2}}\right\} \\
B= & 2 i \sum_{n=1}^{\infty} \frac{n l d \sin n \phi}{n^{2} l^{2}+d^{2}}\left\{2 \frac{K_{1}\left[n^{2} l^{2}+d^{2}\right]^{1 / 2}}{\left[n^{2} l^{2}+d^{2}\right]^{1 / 2}}+K_{0}\left[n^{2} l^{2}+d^{2}\right]^{1 / 2}\right\} \\
C= & K_{0}(d)+\frac{K_{1}(d)}{d}+2 \sum_{n=1}^{\infty}\left\{d^{2} \frac{K_{0}\left[n^{2} l^{2}+d^{2}\right]^{1 / 2}}{n^{2} l^{2}+d^{2}}\right. \\
& \left.-\left(n^{2} l^{2}-d^{2}\right) \frac{K_{1}\left[n^{2} l^{2}+d^{2}\right]^{1 / 2}}{\left[n^{2} l^{2}+d^{2}\right]^{3 / 2}}\right\} \cos n \phi, \\
D= & A-2 \sum_{n=1}^{\infty} K_{0}(n l)(1-\cos n \phi)+\sum_{n=-\infty}^{\infty} K_{0}\left[n^{2} l^{2}+d^{2}\right]^{1 / 2} \\
E= & C-K_{0}(d)-2 \sum_{n=1}^{\infty} K_{0}\left[n^{2} l^{2}+d^{2}\right]^{1 / 2} \cos n \phi .
\end{aligned}
$$

From symmetry the equations for the motion of the zero vortex in the upper row can be written as

$$
\begin{aligned}
& \frac{d}{d t}\left(\overline{\Delta x}_{0}\right)=-A \overline{\Delta y}_{0}+B \Delta x_{0}-C \Delta y_{0}, \\
& \frac{d}{d t}\left(\overline{\Delta y}_{0}\right)=-D \overline{\Delta x}_{0}-B \Delta y_{0}-E \Delta x_{0} .
\end{aligned}
$$

It may be noticed that the second pair of equations is identical to the first in either of two cases, first if $\Delta x_{0}=\overline{\Delta x}_{0}$ and $\Delta y_{0}=-\overline{\Delta y_{0}}$ and second if $\Delta x_{0}=-\overline{\Delta x}_{0}$ and $\Delta y_{0}=\overline{\Delta y}_{0}$. The first case corresponds to symmetrical perturbations with respect to the center line and the 
second case corresponds to antisymmetrical perturbations. In order for the system to be stable, both types of motion must be stable.

$$
\left(\frac{d}{d t}-B\right) \Delta x_{0}=(A-C) \Delta y_{0}, \quad\left(\frac{d}{d t}-B\right) \Delta y_{0}=(D+E) \Delta x_{0}
$$

for the symmetrical case. As $B$ is an imaginary quantity, the condition for stability in this case is that

$$
(A-C)(D+E) \leqq 0 .
$$

For the antisymmetrical perturbations the corresponding stability condition is that

$$
(A+C)(D-E) \leqq 0 .
$$

For the special case where $\phi=\pi$ it is easily seen that the first of these is violated, for from $(26)$ both $(A-C)$ and $(D+E)$ are negative. From this, it follows that the symmetrical vortex system of Figure 4 is unstable.

For the asymmetrical system of Figure 4 it is found by a similar procedure that (25) and (27) and thus (29) and (30) hold if

$$
\begin{aligned}
A= & -2 \sum_{n=1}^{\infty} \frac{K_{1}(n l)}{n l}(1-\cos n \phi) \\
& +2 \sum_{n=0}^{\infty}\left\{\frac{(n+1 / 2)^{2} l^{2}-d^{2}}{R_{n}^{3}} K_{1}\left(R_{n}\right)-\frac{d^{2}}{R_{n}^{2}} K_{0}\left(R_{n}\right)\right\}, \\
B= & 2 i \sum_{n=0}^{\infty} \frac{(n+1 / 2) l d}{R_{n}^{2}}\left\{2 \frac{K_{1}\left(R_{n}\right)}{R_{n}}+K_{0}\left(R_{n}\right)\right\} \sin (n+1 / 2) \phi, \\
C= & 2 \sum_{n=0}^{\infty}\left\{\frac{d^{2}}{R_{n}^{n}} K_{0}\left(R_{n}\right)-\frac{(n+1 / 2)^{2} l^{2}-d^{2}}{R_{n}^{3}} K_{1}\left(R_{n}\right)\right\} \\
& \cdot \cos (n+1 / 2) \phi, \\
D= & A-2 \sum_{n=1}^{\infty} K_{0}(n l)(1-\cos n \phi)+2 \sum_{n=0}^{\infty} K_{0}\left(R_{n}\right), \\
E= & C-2 \sum_{n=0}^{\infty} K_{0}\left(R_{n}\right) \cos (n+1 / 2) \phi,
\end{aligned}
$$

where $R_{n}=\left[(n+1 / 2)^{2} l^{2}+d^{2}\right]^{1 / 2}$. For $\phi=\pi$, the critical case for stability, $C=E=0$. The stability criterion thus becomes $A(\pi) D(\pi)=0$. From (31) it is apparent that this condition will be satisfied for a given value of $l$ for a range of values of $d$ between the limits which 
correspond respectively to $A(\pi)=0$ and $D(\pi)=0$. These limits are given in Table 1 and are shown graphically in Figure 5 . It should be

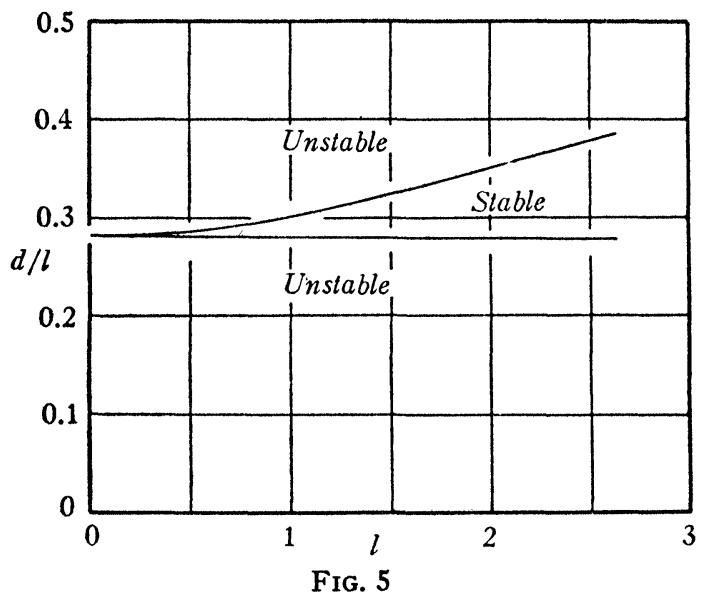

Stability diagram for double vortex rows

TABLE 1

Stable range of values for $d / l$ for asymmetric vortex street

\begin{tabular}{ccc}
\hline$l$ & Upper limit & Lower limit \\
\hline 0 & 0.281 & 0.281 \\
1 & 0.300 & 0.281 \\
2 & 0.350 & 0.280 \\
\hline
\end{tabular}

noted that for small values of $l$, the result that $d / l=0.281$ is exactly that obtained by Kármán in his work with vortex streets behind two-dimensional bluff bodies (see [4, p. 228]).

This calculation shows that stable configurations do exist. These results would be directly applicable to motions in the earth's atmosphere if the width of the vortex street were small compared to the radius of the earth. Unfortunately, this is not so, and the curvature of the shear fields on the edges of the westerlies must be considered. These effects will be considered in the next section.

Stability of rings of vortices. The analysis of the stability of a double ring system of vortices, the system one would use to represent the vortex pattern accompanying the belt of westerly winds, is unfortunately so complex that a successful solution has not yet been 
carried out. The effects of the curvature on the stability of such systems can, however, be estimated from the investigation of the stability of a single ring of vortices. This corresponds to a condition where the shear field to the north of the westerlies is of negligible strength. As the shear field to the north of the westerlies is much weaker than that to the south, this single ring of anticyclonic vortices furnishes a much better picture of the atmospheric motions than the double row of equal vortices investigated in the preceding section.

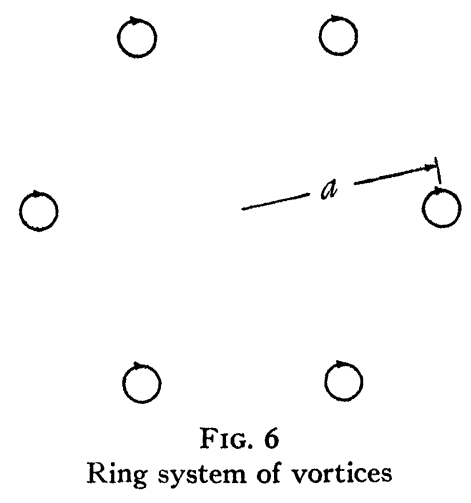

If the shear field north of the westerlies is neglected, a ring of equally strong anticyclones evenly spaced around a latitude circle is the only formation of vortices which will not change its shape with time. The stability of such a system can be investigated approximately by considering the stability of a ring of vortices of the type given by (14). If there are $N$ such vortices in a ring of radius $a$, spaced at equal angles $\tau=2 \pi / N$, as shown in Figure 6 , the surface deflection for such a system in its equilibrium state is

$$
\eta=\sum_{n=1}^{N} K_{0}\left[a^{2}+r^{2}-2 a r \cos (\theta-n \tau)\right]^{1 / 2} .
$$

For the calculation of the velocities of the system it is useful to know the form taken by (16) in polar coordinates. If $v_{r}$ and $v_{\theta}$ are the dimensionless velocities in the radial and tangential directions, respectively, (16) can be written as

$$
v_{r}=-\frac{1}{r} \frac{\partial \eta}{\partial \theta}, \quad v_{\theta}=\frac{\partial \eta}{\partial r} .
$$

From the second of these, the system shown in Figure 6 is seen to have an angular velocity $\Omega$ given by 


$$
\Omega=-(1 / a) \sum_{n=1}^{N-1} K_{1}[2 a \sin (n \tau / 2)] \sin (n \tau / 2) .
$$

If the $n$th vortex is displaced by $\Delta r_{n}$ in the radial direction and by an angle of $\Delta \theta_{n}$ in the tangential direction, the surface deflection in the displaced condition is

$$
\eta=\sum_{n=1}^{N} K_{0}\left[\left(a+\Delta r_{n}\right)^{2}+r^{2}-2\left(a+\Delta r_{n}\right) r \cos \left(\theta-n \tau-\Delta \theta_{n}\right)\right]^{1 / 2}
$$

If the displacements are small, the change in velocity from the equilibrium value given by (34) can be readily calculated. For the $N$ th vortex it is found that

$$
\begin{aligned}
\Delta v_{\tau}= & \frac{d}{d t}\left(\Delta r_{N}\right)=(1 / 2) \sum_{n=1}^{N-1} \Delta r_{n} K_{0}\left(R_{n}\right) \sin n \tau \\
& +\sum_{n=1}^{N-1} a\left(\Delta \theta_{n}-\Delta \theta_{N}\right)\left\{\frac{K_{1}\left(R_{n}\right)}{R_{n}}+K_{0}\left(R_{n}\right) \cos ^{2}(n \tau / 2)\right\}, \\
\Delta v_{\theta}= & a \frac{d}{d t}\left(\Delta \theta_{N}\right)+\Omega \Delta r_{N} \\
= & \Delta r_{N} \sum_{n=1}^{N-1}\left\{K_{0}\left(R_{n}\right) \sin ^{2}(n \tau / 2)-\frac{K_{1}\left(R_{n}\right)}{R_{n}} \cos n \tau\right\} \\
& +\sum_{n=1}^{N-1}\left[\Delta r_{n}\left\{\frac{K_{1}\left(R_{n}\right)}{R_{n}}+K_{0}\left(R_{n}\right) \sin ^{2}(n \tau / 2)\right\}\right. \\
& \left.+(1 / 2) a \Delta \theta_{n} K_{0}\left(R_{n}\right) \sin n \tau\right]
\end{aligned}
$$

where $R_{n}=2 a \sin (n \tau / 2)$. Similar expressions for the velocities of the other vortices could be written from symmetry. These would form a set of simultaneous differential equations for the displacements.

If the $N$ equations in each of the two sets indicated in (36) are added, it is seen that

$$
\begin{aligned}
\frac{d}{d t}\left\{\sum_{n=1}^{N} \Delta r_{n}\right\} & =0 \\
\frac{d}{d t}\left\{\sum_{n=1}^{N} \Delta \theta_{n}\right\}= & \left\{\sum_{n=1}^{N} \Delta r_{n}\right\} \\
& \cdot\left\{\sum_{n=1}^{N-1}\left[2 \sin ^{2}(n \tau / 2) K_{0}\left(R_{n}\right)+(1 / a) \sin (n \tau / 2) K_{1}\left(R_{n}\right)\right]\right\}
\end{aligned}
$$


If the system is initially in equilibrium so that initially $\Delta r_{n}=\Delta \theta_{n}=0$, then (37) shows that

$$
\sum_{n=1}^{N} \Delta r_{n}=\sum_{n=1}^{N} \Delta \theta_{n}=0 .
$$

These results correspond to the similar equations for two-dimensional line vortices which state that the impulse of a system having no external forces remains constant (see $[4$, p. 220]).

The method of harmonic analysis used in the preceding section can also be used to advantage in the solution of (36). If $\Delta r_{n}=\Delta r_{N} e^{i n \phi}$ and $\Delta \theta_{n}=\Delta \theta_{N} e^{i n \phi}$ where $\phi$ is a member of the series $2 \pi / N, 4 \pi / N, \cdots$, $(N-1) 2 \pi / N, 2 \pi$, then (36) can be written as

$$
\begin{aligned}
\frac{d}{d t}\left(\Delta r_{N}\right) & =A \Delta r_{N}-B a \Delta \theta_{N}, \\
a \frac{d}{d t}\left(\Delta \theta_{N}\right) & =C \Delta r_{N}+A a \Delta \theta_{N}
\end{aligned}
$$

where

$$
\begin{aligned}
& A=(1 / 2) \sum_{n=1}^{N-1} e^{i n \phi} \sin n \tau K_{0}\left(R_{n}\right), \\
& B=\sum_{n=1}^{N-1}\left(1-e^{i n \phi}\right)\left\{\frac{K_{1}\left(R_{n}\right)}{R_{n}}+\cos ^{2}(n \tau / 2) K_{0}\left(R_{n}\right)\right\}, \\
& C=\sum_{n=1}^{N-1}\left\{\left(1+e^{i n \phi}\right) \sin ^{2}(n \tau / 2) K_{0}\left(R_{n}\right)\right. \\
& \left.\quad+\left(1-2 \cos n \tau+e^{i n \phi}\right) \frac{K_{1}\left(R_{n}\right)}{R_{n}}\right\} .
\end{aligned}
$$

Since $A$ is a purely imaginary quantity for any of the specified values of $\phi$, the condition that the system be stable imposed by (39) is that

$$
B C \geqq 0 .
$$

From (40) it may be seen that $B$ is never negative and that $C$ is always positive for $N=2,3,4,5$, or 6 . For $N=7, C$ is positive if $a>71$. For $N>7, C$ is always negative for one or more of the values of $\phi$. A value of $a>71$ corresponds either to disturbances of such great wave length or to motions of such a shallow layer of air in the earth's atmosphere that it probably is of no significance. The results of this calculation may then be summarized by the statement that six or 
less equal vortices placed at the corners of a regular polygon form a stable vortex formation. This result is in agreement with the work of J. J. Thomson who investigated the stability of similar formations of two-dimensional line vortices in a frictionless incompressible fluid (see $[6$, p. 94]). The effect of the curvature of the row of vortices is thus seen to be stabilizing providing the number of the vortices in the ring is small enough.

As a single ring is stable, the effect of the shear field to the north of the westerlies can roughly be taken into account by placing a fixed vortex at the pole as in Figure 7. The effect of such a vortex is to

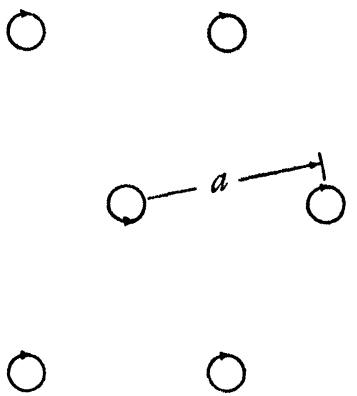

FIG. 7

Ring system of vortices with polar cyclone

change the rate of rotation of the system and to add additional turns to (40). If $P$ is the ratio of polar (cyclonic) vorticity to the anticyclonic vorticity, then (39) still holds if

$$
C=C(P=0)-N P\left\{K_{0}(a)+2 \frac{K_{1}(a)}{a}\right\} .
$$

The fixed polar vortex is thus seen to cause a decrease in stability and if $P$ is large enough the motion becomes unstable. As $P$ is increased from zero it is found that the maximum number of vortices which form a stable formation diminishes from six to five, to four, to three and then to zero.

Vortex motion on a rotating sphere. All the calculations discussed in the preceding sections have neglected the effect of the variation of the Coriolis acceleration with latitude, that is, they correspond to conditions on a rotating disc rather than on a rotating sphere. In this section vortex motion in an atmosphere on a rotating sphere will be discussed. 
In terms of polar coordinates on a sphere rotating with an angular velocity $\omega$ with $\theta$ being the angle from the north pole and $\phi$ being the longitudinal angle, the equation representing the conservation of angular momentum and corresponding to (4) is

$$
\frac{D}{D t}\left\{\frac{\zeta+2 \omega \cos \theta}{h}\right\}=0
$$

where $\zeta$ is the radial component of the curl of the velocity vector. If $u$ is the velocity to the east and $v$ is the velocity to the north, the expression for $\zeta$ is

$$
\zeta=\frac{1}{a \sin \theta}\left\{\frac{\partial}{\partial \theta}(u \sin \theta)+\frac{\partial v}{\partial \phi}\right\} .
$$

This principle will be used to investigate the velocity distribution which would accompany a vortex with its core at the north pole. For this case it is permissible to assume symmetry about the polar axis with $v$ being zero in the steady state and $u$ and $h$ being functions only of $\theta$.

If the motion started from rest with the atmosphere having a uniform depth, $h_{0}$, (43) can be integrated to give

$$
\frac{\zeta+2 \omega \cos \theta}{h}=\frac{2 \omega}{h_{0}} \cos \theta_{0}
$$

where $\theta_{0}$ is the colatitude at which the particle originated. As the mass of the fluid must be conserved,

$$
\int_{\theta}^{\pi} h_{0} \sin \theta d \theta=\int_{\theta}^{\pi} h \sin \theta d \theta
$$

From this,

$$
\cos \theta_{0}=-1+\int_{\theta}^{\pi} \frac{h}{h_{0}} \sin \theta d \theta,
$$

and the vorticity at any latitude is thus

$$
\zeta=2 \omega\left\{-\frac{h}{h_{0}}-\cos \theta+\frac{h}{h_{0}} \int_{\theta}^{\pi} \frac{h}{h_{0}} \sin \theta d \theta\right\} .
$$

The equation of motion is

$$
\frac{1}{a} \cot \theta u^{2}+2 \omega \cos \theta u=\frac{g}{a} \frac{d h}{d \theta}
$$


and from (44)

$$
\zeta=\frac{1}{a \sin \theta} \frac{d}{d \theta}(u \sin \theta)
$$

Equations (48), (49), and (50) must be solved simultaneously for $u$, $h$, and $\zeta$. An exact solution is extremely difficult, but an approximate solution can be obtained if the centrifugal acceleration is neglected in (49) and if the depth of the atmosphere is only slightly different from the equilibrium value $h_{0}$.

If the centrifugal acceleration is neglected, these equations may be combined to give

$$
\frac{1}{\sin \theta} \frac{d}{d \theta}\left\{\tan \theta \frac{d \eta}{d \theta}\right\}=\alpha^{2}\left\{\eta \cos \theta+(1+\eta) \int_{\theta}^{\pi} \eta \sin \theta d \theta\right\}
$$

where

$$
\alpha^{2}=4 \omega^{2} a^{2} /\left(g h_{0}\right) \text { and } \eta=\left(h-h_{0}\right) / h_{0} .
$$

Since $\eta$ is small compared to unity, (51) may be linearized by replacing the factor $1+\eta$ by 1 . If a new independent variable, $\mu=\cos \theta$, is introduced, (51) can be then written in the form

$$
\frac{d^{2}}{d \mu^{2}}\left\{\frac{1-\mu^{2}}{\mu} \frac{d \eta}{d \mu}\right\}-\alpha^{2}\left\{\mu \frac{d \eta}{d \mu}+2 \eta\right\}=0 .
$$

This equation can be integrated once, and it then becomes

$$
\mu^{2} \frac{d}{d \mu}\left\{\frac{1-\mu^{2}}{\mu^{2}} \frac{d \eta}{d \mu}\right\}-\alpha^{2} \mu^{2} \eta=C
$$

where $C$ is an arbitrary constant. This equation (with $C=0$ ) is identical with an equation used in the development of the dynamical theory of the long period fortnightly and semiannual tides (see $[4, \mathrm{p}$. 334]). The desired solution of (53) is finite except for $\mu=1$ and satisfies the continuity principle; that is,

$$
\int_{-1}^{1} \eta d \mu=0
$$

For standard atmospheric conditions with the sea level pressure and density being $1013.3 \mathrm{mb}$ and $1.276 \times 10^{3} \mathrm{gm} / \mathrm{cc}$, respectively, the constant $\alpha^{2}$ has the value 10.9. The velocity distribution obtained from the solution of (53) for this value of $\alpha^{2}$ which satisfies the given boundary conditions is plotted in Figure 8 together with the cor- 
responding solution for the velocity distribution for a vortex on a rotating disc as given by (15).

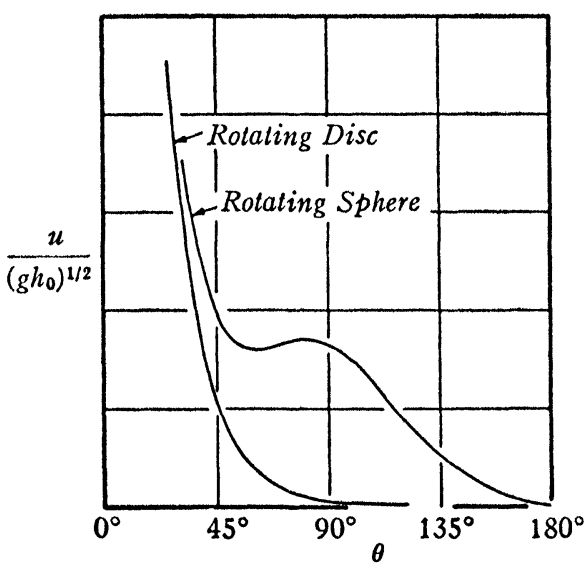

FIG. 8

Velocity distribution for a polar vortex on a rotating sphere

It would be desirable to extend these calculations to obtain the velocity distribution for a vortex arbitrarily placed on a sphere and to use this result to discuss the stability of vortex formations on a sphere. It may be seen from this calculation of the velocity field of a polar vortex that such an investigation would be very difficult to carry out satisfactorily.

Concluding remarks. It has been shown that a ring of equal anticyclones formed by the "rolling-up" of the shear field south of the westerlies is stable provided that the number of anticyclones is not more than six and that the shear field north of the westerlies is not strong. The most unsatisfactory feature of this dynamic picture is that if it were strictly true, the anticyclones would have a small velocity to the west; however it is apparent that the effect of the vorticity north of the westerlies would be to decrease this velocity. The "vortex street," for which the cyclonic and anticyclonic vorticities are equal, has a velocity to the east; so that an analysis which properly took into account the relatively small amount of cyclonic vorticity to the north of the westerlies might be expected to show stable systems which are practically stationary. This is in rough agreement with the idea that the position of such systems ought to be determined primarily by thermodynamic considerations, so that the 
stable vortex formations found in the atmosphere might be expected to be those that are stationary.

Since the cyclonic vorticity does not normally show concentrations like the Pacific and the Azores anticyclones-the Aleutian and Icelandic lows apparently being surface phenomena caused by the passage of large numbers of low level-storms-the representation of the cyclonic vorticity by a single polar vortex is probably a fairly good approximation. As this vortex was seen to cause a decrease in the number of anticyclones that could form stable formations, the maximum number which can exist in the atmosphere is probably less than six with the most stable formation of three anticyclones being the most probable. There is about $120^{\circ}$ of longitude separating the mean positions of the Pacific and the Azores highs, and there is some evidence of the existence of a similar system equally distant from these two over Asia, but this evidence is far from conclusive.

These stability calculations were made with vortices on a rotating disc rather than on a rotating sphere, and the possibility of coupling between northern and southern hemispheric systems was neglected. It is not possible to state "a priori" whether or not these modifications are important; however it is believed that their effect is not large.

\section{REFERENCES}

1. H. J. Stewart, Proc. Nat. Acad. Sci. U.S.A. vol. 26 (1940) p. 604.

2. C. G. Rosby, Journal of Marine Research vol. 2 (1939) p. 38.

3. B. Haurwitz, Journal of Marine Research vol. 3 (1940) p. 255.

4. H. Lamb, Hydrodynamics, 6th edition, Cambridge University Press, London, 1932.

5. A. Grey, G. B. Mathews and T. M. MacRobert, Bessel Functions, London, 1931.

6. J. J. Thomson, Motion of Vortex Rings, London, 1883.

California Institute of Technology 